\title{
Do not drink and lyse: alcohol intoxication increases fibrinolysis shutdown in injured patients
}

\author{
Gregory R. Stettler ${ }^{1}$ Ernest E. Moore ${ }^{1,2} \cdot$ Geoffrey R. Nunns ${ }^{1} \cdot$ Hunter B. Moore ${ }^{1} \cdot$ Benjamin R. Huebner ${ }^{1}$. \\ Christopher C. Silliman ${ }^{1,3,4} \cdot$ Anirban Banerjee ${ }^{1} \cdot$ Angela Sauaia $^{1,5}$
}

Received: 25 October 2019 / Accepted: 11 February 2020 / Published online: 10 March 2020

○) Springer-Verlag GmbH Germany, part of Springer Nature 2020

\begin{abstract}
Introduction High alcohol consumption has been associated with decreased fibrinolysis and enhanced thrombosis risk in cardiovascular disease. In trauma, alcohol has been associated with poor clot formation; however, its effect on fibrinolysis has not been fully investigated. We assessed the association of blood alcohol levels and fibrinolysis in trauma activation patients. Methods We queried our prospective registry of trauma activations from 2014 to 2016. Associations between viscoelastic measurements [rapid thrombelastography (rTEG)] and blood alcohol level (BAL) were determined and adjusted for confounders by a multinomial logistic regression. Lysis phenotypes were defined by the \% lysis in $30 \mathrm{~min}$ (LY30) as follows: hyperfibrinolysis $\geq 3 \%$, physiologic $0.9-2.9 \%$, and fibrinolysis shutdown $<0.9 \%$.

Results Overall, 191 (43.8\%) had BAL measured. There were 65 (34\%) patients that had no detectable BAL, 32 (16.8\%) had BAL of $10-150 \mathrm{mg} / \mathrm{dL}$, and $94(49.2 \%)$ patients had BAL $>150 \mathrm{mg} / \mathrm{dL}$. BAL had a moderate, but significant inverse correlation with LY30 (Rho $=-0.315, p<0.001$ ), while there were no significant correlations between BAL and other TEG values. The distribution of fibrinolysis phenotypes varied significantly by BAL levels $(p<0.009$, with high BAL having more shutdown and less hyperfibrinolysis than the other two BAL level groups. Multinomial logistic regression showed that after adjustment for confounders, BAL levels $>150 \mathrm{mg} / \mathrm{dL}$ were independently associated with a threefold increase in the odds of shutdown compared to undetectable BAL (OR 3.37, 95\% CI 1.04-8.05, $p=0.006$ ). High BAL was also significantly associated with higher odds of shutdown compared to low BAL (OR 2.63, 95\% CI 1.15-6.06). Compared to physiologic fibrinolysis, fibrinolysis shutdown was associated with increased mortality (OR 2.87, 95\% CI 1.41-5.83) and VFD $<28$ (OR $2.54,95 \%$ CI $1.47-4.39)$.

Conclusion In the injured patient, high blood alcohol levels are associated with increased incidence of fibrinolysis shutdown. This finding has implications for postinjury hemostatic resuscitation as these patients may be harmed by anti-fibrinolytics. Further research is needed to assess whether the association with fibrinolysis is modified by the chronicity and type of alcohol consumed and whether anti-fibrinolytic therapy in intoxicated patients produces adverse effects.
\end{abstract}

Keywords Trauma-induced coagulopathy $\cdot$ Alcohol $\cdot$ Alcohol intoxication $\cdot$ Fibrinolysis shutdown

\section{Introduction}

Gregory R. Stettler

gregory.stettler@ucdenver.edu

1 Department of Surgery, University of Colorado Denver, 12631 E. 17th Ave., C302, Aurora, CO 80045, USA

2 Denver Health Medical Center, Aurora, USA

3 Department of Pediatrics, University of Colorado Denver, Aurora, USA

4 Vitalant Research Institute, Denver, USA

5 School of Public Health, University of Colorado, Aurora, USA
Alcohol intoxication leads to metabolic and physiologic derangements that complicate the care of intoxicated trauma patients compared to their non-intoxicated counterparts [1]. These physiologic changes include impaired cardiovascular function, blunting of catecholamine release leading to inadequate oxygen delivery to tissue and metabolic uncoupling, modulation of the innate immune system, and alterations in blood coagulation [1-8].

Acute and chronic alcohol consumption can have varying effects, and distinct, sometimes opposite, effects on 
coagulation following alcohol intoxication have been described in the literature. Some have described hypocoagulability in the intoxicated patient as evidenced by prolonged initiation of clot formation and decreased dynamics of clot formation resulting in a reduced risk of venous thrombotic events (VTE) in trauma [3, 4]. Conversely, others have established a link between alcohol intoxication and increased risk of thrombotic complications through elevated levels of plasminogen activator inhibitor-1 (PAI-1), the primary inhibitor of fibrinolysis [2, 9]. This differential risk of adverse effects appears to be related to the amount of and timing within which ethanol is consumed $[10,11]$. While there is a decrease in fibrinogen and platelets reported with excess alcohol consumption [12], there is also a reported increase in coagulation Factor VII, Factor VIII, and PAI-1 in the acutely intoxicated $[9,12,13]$. On the other hand, Factor VII has been shown to decrease in chronic alcohol use, likely from liver disease and synthetic dysfunction [14]. Furthermore, there is a greater increase in PAI-1 release compared to tissue plasminogen activator (tPA) in those who consume a larger amount of alcohol, thereby increasing the PAI-1:tPA ratio [12].

Elevated levels of PAI-1 are associated with increased fibrinolysis shutdown, which is the most common fibrinolytic phenotype in the injured population and is associated with an increased mortality due to macro- and microthrombotic complications such as venous thrombotic events (VTE) and multiple organ failure (MOF) [15]. Fibrinolysis shutdown has previously been identified as the most common fibrinolytic phenotype following injury and is also associated with increased mortality compared to physiologic fibrinolysis, often due to multiple organ failure [15-17] and has most commonly been measured by thrombelastography (TEG), a viscoelastic assay that provides a comprehensive assessment of clot formation and clot remodeling and degradation. Therefore, we hypothesize that alcohol intoxication is associated with an increased incidence of fibrinolysis shutdown on thrombelastography.

\section{Methods}

\section{Study design}

Our prospective Trauma Activation Protocol (TAP) registry includes all adult ( $\geq 18$ years old) patients who met the criteria for the highest level of trauma team activation from 2014 to 2016 at the Ernest E Moore Shock Trauma Center at Denver Health (DHMC), Denver, CO, an American College of Surgeons verified and state-certified Level 1 trauma center. Exclusion criteria were unsalvageable injuries (defined by patients in asystole at emergency department arrival), isolated gunshot wounds to the head, pregnancy, documented chronic liver disease, or a known coagulation disorder. A rapid thrombelastogram (r-TEG) was run on whole blood from all of these patients under waiver of consent at the scene or immediately upon arrival less than $1 \mathrm{~h}$ postinjury. This clinical study was approved by the Colorado Multiple Institutional Review Board (COMIRB).

Clinical data were collected by trained research professional assistants (PRAs) and included demographic characteristics, injury severity, physiologic derangement, transfusions, and outcomes [death, intensive care-free days (ICUFD), ventilator-free days (VFD)]. Severe traumatic brain injury was defined as an Abbreviated Injury Scale (AIS) for Head $\geq 3$. The New Injury Severity Score (NISS) measured injury severity. Massive transfusion was defined as greater than 10 units of red blood cells (RBC) or death within $6 \mathrm{~h}$ postinjury as we have found this definition to be related to adverse outcomes $[18,19]$. Further, the inclusion of death within $6 \mathrm{~h}$ was to minimize survivor bias (i.e., nonsurvivors did not have the "opportunity" to receive transfusions) $[18,19]$. The protocol for massive transfusion of blood products has been described previously and includes initial empiric blood component therapy (FFP:RBC in 1:2 ratio) [20] followed by r-TEG-guided hemostatic resuscitation base on ACT [s], angle $\left({ }^{\circ}\right)$, maximum amplitude [MA $(\mathrm{mm})$ ], and lysis $30 \mathrm{~min}$ after MA (LY30 [\%] [21].

\section{Blood samples from trauma patients}

Samples were collected during trauma activations within $1 \mathrm{~h}$ of injury in the field or emergency department (ED) in tubes containing $3.2 \%$ citrate. All blood samples were obtained prior to administration of plasma or tranexamic acid (TXA). Determination of blood alcohol level (BAL) was requested at the attending physician's discretion. The lower limit of detection for BAL is $10 \mathrm{mg} / \mathrm{dL}(0.1 \mathrm{~g} / \mathrm{L})$.

\section{Rapid TEG}

A team of trained professional research assistants completed the viscoelastic assays. Citrated blood samples were analyzed using the TEG 5000 Thrombelastography Hemostasis Analyzer (Haemonetics, Niles, IL, USA) as previously described [22]. The rapid TEG (activated by tissue factor and kaolin) was employed and the following indices were obtained from the tracings of the TEG: activated clotting time [ACT (s)], angle $\left({ }^{\circ}\right)$, maximum amplitude [MA (mm)], and lysis $30 \mathrm{~min}$ after MA [LY30 (\%)]. Definitions for fibrinolysis phenotypes by rTEG were shutdown $(\leq 0.9 \%)$, physiologic $(>0.9-\leq 2.9 \%)$, and hyperfibrinolysis $(\geq 3 \%)$ as previously described [15]. 


\section{Statistical analysis}

SAS version 9.4 (SAS Institute, Inc. Cary, NC, USA) was used for statistical analysis. Non-normally distributed variables were expressed as median and interquartile range (IQR) and the Wilcoxon non-parametric test or the Kruskal-Wallis test was used for continuous variables. Normally distributed variables were presented as mean (standard deviation, SD). Fisher's exact test was used for categorical variables. The non-parametric Spearman Rho test was used for correlations. Receiver operator characteristic (ROC) curve analysis was done to determine the predictability of BAL to predict fibrinolysis phenotypes. Multinomial logistic regression models were used to assess the independent effect of BAL on the three lysis phenotypes. Model fit was assessed via deviance and the Pearson goodness of fit tests (higher $p$ values reflect better fit). To minimize selection bias due to missing BAL, we conducted a sensitivity analysis including the patients without a measured BAL as an additional, separate category. All tests were two tailed with significance declared at $p<0.05$.

\section{Results}

Of 436 consecutive trauma activation patients enrolled between 2014 and 2016, 191 (43.8\%) had blood alcohol levels (BAL) measured. Table 1 illustrates differences in patients that had a BAL measured and those that did not. Compared to patients for whom BALs was not obtained, those with BALs measured were more likely to have blunt trauma $(68.1 \%$ vs $40.8 \%, p<0.001)$ and TBI $(21.5 \%$ vs. $12.6 \%, p=0.01)$, had increased incidence of isolated TBI $(12.57 \%$ vs $6.12 \%, p=0.02)$, had slightly, but significantly lower INR (1.2 (0.4) vs. $1.3(0.5), p=0.01)$, were less likely receive antifibrinolytics in the form of tranexamic acid (4.19\% vs $9.39 \%, p=0.36)$ and were less likely to undergo a massive transfusion $(4.19 \%$ vs $20.82 \%, p<0.001)$. Other demographic and injury characteristics were similar. The median BAL was $150 \mathrm{mg} / \mathrm{dL}(1.5 \mathrm{~g} / \mathrm{L})$ (IQR: 0-251); therefore, we used this cutoff to define the high BAL group $(>150 \mathrm{mg} / \mathrm{dL}$ or $>1.5 \mathrm{~g} / \mathrm{L})$ versus low BAL $(10-150 \mathrm{mg} / \mathrm{dL}$ or $0.1-1.5 \mathrm{~g} / \mathrm{L})$. The third category was undetectable BAL $(<10 \mathrm{mg} / \mathrm{dL}$ or $<0.1 \mathrm{~g} / \mathrm{L})$.

\section{Blood alcohol levels associated with clinical and laboratory risk factors}

Overall, 65 (34\%) patients had no detectable BAL, 32 $(16.8 \%)$ had BAL of $10-150 \mathrm{mg} / \mathrm{dL}(0.1-1.5 \mathrm{~g} / \mathrm{L})$, and 94 (49.2\%) patients had BAL $>150 \mathrm{mg} / \mathrm{dL}(>1.5 \mathrm{~g} / \mathrm{L})$. Characteristics of patients stratified by BAL categories are depicted in Table 2. Patients with a high BAL had lower admission systolic blood pressure (SBP), increased base deficit (BD), and decreased LY30 ( $p<0.001$ for all) (Table 1). BAL had a moderate, but significant inverse linear correlation with LY30 (Rho $=-0.315, p<0.001$ ), while there were no significant correlations between BAL and other TEG values (ACT, angle, MA) (Table 3).

\section{Blood alcohol level and fibrinolysis phenotypes in injured patients}

The distribution of fibrinolysis phenotypes varied significantly by BAL levels (Fig. 1, $p=0.009$ ), with high BAL having more shutdown and less hyperfibrinolysis than the other two BAL groups.

Multinomial logistic regression for the three-category lysisdependent variable (hyperfibrinolysis, shutdown, and physiologic serving as the reference group) showed that, after adjustment for age, blunt mechanism, NISS, admission GCS, and SBP, BAL levels $>150 \mathrm{mg} / \mathrm{dL}(\gg 1.5 \mathrm{~g} / \mathrm{L}$ ) were independently associated with a threefold increase in the odds of shutdown compared to undetectable BAL (OR 3.37, 95\% CI 1.04-8.05, $p=0.006$ ). High BAL was also significantly associated with higher odds of shutdown compared to low BAL (OR 2.63, 95\% CI 1.15-6.06) as shown in Table 4. Low BAL was not significantly associated with abnormal lysis. Hyperfibrinolysis was not associated with either high or low BAL. A BAL $>150$ is an independent predictor of fibrinolysis shutdown (AUROC $0.76,95 \% 0.68-0.85$ ).

In the sensitivity analysis, we added the patients for whom BAL was not obtained, adjusted for the same covariates as above. High BAL remained independently associated with shutdown compared to undetectable BAL (OR 2.93, 95\% CI 1.12-7.67) and compared to untested patients (OR 2.05, 95\% CI 1.07-3.93).

In binomial multiple logistic regression, high BAL did not independently affect mortality $(p=0.62)$, VFD $<28(p=0.53)$, or ICUFD $<25$ days $(p=0.94)$, nor did it modify the association between fibrinolysis phenotype and these outcomes ( $p>0.05$ for all interactions). We confirmed previous results that compared to physiologic fibrinolysis, both abnormal fibrinolysis phenotypes were associated with increased mortality (hyperfibrinolysis OR 2.27, 95\% CI 1.13-4.58; shutdown OR 2.87, 95\% CI 1.41-5.83) and VFD <28 (hyperfibrinolysis OR 1.95, 95\% CI 1.20-3.17; shutdown OR 2.54, 95\% CI 1.47-4.39). The number of patients with high BAL who died $(n=6)$ and the number of VTE in these patients $(n=2)$ were too small to allow reliable analyses within these subgroups. 
Table 1 Demographic characteristics of patients with and without ETOH measured

\begin{tabular}{|c|c|c|c|c|c|c|c|}
\hline \multirow[t]{2}{*}{ Variables } & \multicolumn{3}{|c|}{ ETOH not measured } & \multicolumn{3}{|c|}{ ETOH measured } & \multirow[t]{2}{*}{$p$ value } \\
\hline & Median & Lower quartile & Upper quartile & Median & Lower quartile & Upper quartile & \\
\hline Age & 31.8 & 25.8 & 48 & 34 & 26.1 & 47.4 & 0.443 \\
\hline ED SBP & 115 & 90 & 139 & 110 & 90 & 139 & 0.84 \\
\hline ED HR & 100 & 78 & 120 & 102 & 80 & 116 & 0.9 \\
\hline ED GCS & 15 & 9 & 15 & 14 & 4 & 15 & $<0.001$ \\
\hline ED temp $\left({ }^{\circ} \mathrm{C}\right)$ & 36.6 & 36.3 & 36.9 & 36.5 & 36.2 & 36.8 & 0.017 \\
\hline BMI & 26.4 & 23.9 & 30.6 & 26.1 & 23.5 & 29.7 & 0.41 \\
\hline Blunt mechanism & $40.82 \%$ & & & $68.06 \%$ & & & $<0.001$ \\
\hline NISS & 22 & 9 & 38 & 17 & 6 & 34 & 0.14 \\
\hline Max AIS head and neck & 0 & 0 & 2 & 0 & 0 & 3 & 0.003 \\
\hline Max AIS chest & 0 & 0 & 3 & 0 & 0 & 3 & 0.73 \\
\hline Max AIS abdomen and pelvis & 0 & 0 & 3 & 0 & 0 & 2 & 0.11 \\
\hline Max AIS extremities & 0 & 0 & 3 & 0 & 0 & 2 & 0.83 \\
\hline Positive FAST & $25.59 \%$ & & & $13.79 \%$ & & & 0.001 \\
\hline TBI & $12.65 \%$ & & & $21.47 \%$ & & & 0.014 \\
\hline Isolated TBI & $6.12 \%$ & & & $12.57 \%$ & & & 0.014 \\
\hline Massive transfusion & $20.82 \%$ & & & $4.19 \%$ & & & $<.001$ \\
\hline Death & $17.57 \%$ & & & $31.15 \%$ & & & 0.027 \\
\hline Lactate & 4.3 & 2.9 & 7.5 & 3.7 & 2.6 & 5.3 & 0.012 \\
\hline Base deficit & -6 & -10 & -3 & -7 & -11 & -4 & 0.65 \\
\hline Platelet count & 257 & 202.5 & 309 & 259.5 & 205 & 311 & 0.39 \\
\hline INR & 1.1 & 1 & 1.3 & 1.1 & 1 & 1.3 & 0.021 \\
\hline r-TEG ACT (s) & 121 & 113 & 136 & 121 & 113 & 128 & 0.022 \\
\hline r-TEG angle (deg) & 71.6 & 64.5 & 75.4 & 70.9 & 67 & 75.2 & 0.35 \\
\hline r-TEG MA (mm) & 62 & 55.5 & 66 & 62.5 & 57.5 & 66 & 0.3 \\
\hline r-TEG LY30 (\%) & 1.9 & 1 & 3.4 & 1.9 & 0.9 & 3.2 & 0.38 \\
\hline Anti-fibrinolytics & $9.39 \%$ & & & $4.19 \%$ & & & 0.036 \\
\hline Length of stay (LOS) & 6 & 1.5 & 12 & 6 & 2 & 13 & 0.2 \\
\hline VFD & 26.5 & 17 & 28 & 26 & 23 & 28 & 0.45 \\
\hline ICUFD & 24 & 15 & 28 & 25 & 20 & 27 & 0.19 \\
\hline
\end{tabular}

Continuous variables are presented as median and interquartile range while categorical variables are presented as percent

The ${ }^{*} \chi^{2}$ test was used for categorical variables and Wilcoxon test for continuous variables

$B A L$ blood alcohol level, $T B I$ traumatic brain injury, $B M I$ body mass index, NISS New Injury Severity Score, $E D$ emergency department, $S B P$ systolic blood pressure, $H R$ heart rate, GCS Glasgow Coma Scale, INR International Normalized Ratio, TEG thrombelastography, ACT activated clotting time, MA maximum amplitude, $L Y 30$ lysis $30 \mathrm{~min}$ after MA is achieved, VFD ventilator-free days, ICUFD intensive care unit-free days

\section{Discussion}

The distribution of fibrinolysis phenotypes in intoxicated trauma patients was shifted toward an increased prevalence of fibrinolysis shutdown compared to patients with no detectable alcohol. At the same time, this did not result in an increased risk of death. Previous studies have indicated a number of perturbations in viscoelastic-based measurements of blood clotting in an intoxicated and injured patient [3, 4]. Two previous studies have each shown that the time to clot formation and rate of clot propagation are impaired in intoxicated patients. Our study does not show significant increases in the time to clot formation as measured by the activated clotting time (ACT) in intoxicated patients or the rate of clot propagation. An explanation could be the type of activator used for the TEG assays. Both previous studies used kaolinactivated TEGs while our institution uses rapid TEGs, which are activated with tissue factor as well as kaolin.

Our results are consistent with findings in a recent study [3] suggesting that major adverse outcomes (need for massive transfusion and death) are not significantly affected by acute alcohol intoxication. Howard et al. [3] showed no difference in rates of transfusion and death in those who were intoxicated, specifically, the rates of death and massive transfusion are similar regardless of BAL class for each fibrinolysis phenotype. Overall, we found that patients who 
Table 2 Demographic characteristics by alcohol intoxication status and association with clinical and laboratory risk factors

\begin{tabular}{|c|c|c|c|c|}
\hline \multirow[t]{2}{*}{ Variables } & \multirow{2}{*}{$\begin{array}{l}\text { BAL } 0 \mathrm{mg} / \mathrm{dL} \\
n=65\end{array}$} & \multirow{2}{*}{$\begin{array}{l}\mathrm{BAL}>0-150 \mathrm{mg} / \\
\mathrm{dL} \text { or }>0-1.5 \mathrm{~g} / \mathrm{L} \\
n=32\end{array}$} & \multirow{2}{*}{$\begin{array}{l}\text { BAL }>150 \mathrm{mg} / \mathrm{dL} \\
n=94\end{array}$} & \multirow[b]{2}{*}{$p$ value } \\
\hline & & & & \\
\hline Age & $35.2(25.6$ to 47.9$)$ & $29.5(23.7$ to 41.3$)$ & $37.1(28.6$ to 47.3$)$ & 0.085 \\
\hline Male & $76.92 \%$ & $78.13 \%$ & $81.91 \%$ & 0.063 \\
\hline ED SBP & $120(102$ to 140$)$ & $100(80$ to 126$)$ & $110(90$ to 138$)$ & $<0.001$ \\
\hline ED HR & 103 (83 to 116$)$ & 103 (78 to 112.5$)$ & $100(77$ to 115$)$ & 0.93 \\
\hline ED GCS & $14(4$ to 15$)$ & 15 (10 to 15$)$ & $14(3$ to 15$)$ & 0.11 \\
\hline ED temp $\left({ }^{\circ} \mathrm{C}\right)$ & 36.5 (36.3 to 36.8$)$ & $36.6(36$ to 36.8$)$ & 36.5 (36.1 to 36.8$)$ & 0.67 \\
\hline BMI & $26.1(24.2$ to 29.1$)$ & $25.4(21.8$ to 29.3$)$ & $26.2(23.7$ to 29.8$)$ & 0.66 \\
\hline Blunt mechanism & $67.69 \%$ & $56.25 \%$ & $72.34 \%$ & 0.44 \\
\hline NISS & 19.5 (6 to 42.5$)$ & 16.5 (8 to 27$)$ & $17(5$ to 34$)$ & 0.4 \\
\hline Max AIS head and neck & $0(0$ to 3$)$ & $0(0$ to 1.5$)$ & $0.5(0$ to 3$)$ & 0.24 \\
\hline Max AIS chest & $1(0$ to 3$)$ & $0(0$ to 3$)$ & $1.5(0$ to 3$)$ & 0.77 \\
\hline Max AIS abdomen and pelvis & $0(0$ to 2$)$ & $0(0$ to 2$)$ & $0(0$ to 2$)$ & 0.34 \\
\hline Max AIS extremities & $0(0$ to 2$)$ & $2(0$ to 3$)$ & $0(0$ to 2$)$ & 0.16 \\
\hline Positive FAST & $18.03 \%$ & $7.41 \%$ & $12.79 \%$ & 0.46 \\
\hline TBI & $21.54 \%$ & $15.6 \% 3$ & $23.40 \%$ & 0.27 \\
\hline Isolated TBI & $12.31 \%$ & $9.38 \%$ & $13.38 \%$ & 0.74 \\
\hline Massive transfusion & $6.15 \%$ & $6.25 \%$ & $2.13 \%$ & 0.72 \\
\hline Death & $15.63 \%$ & $9.38 \%$ & $6.45 \%$ & 0.2 \\
\hline Lactate & $3(2.1$ to 4.6$)$ & $3.8(2.7$ to 5.5$)$ & 4.1 (3 to 5.1$)$ & 0.09 \\
\hline Base deficit & $-5(-8$ to -1$)$ & $-7.6(-11$ to -5$)$ & $-7(-11$ to -5$)$ & 0.001 \\
\hline Platelet count & 268 (207 to 310$)$ & 264 (216 to 332$)$ & 252 (201 to 304$)$ & 0.59 \\
\hline INR & 1.1 (1 to 1.2$)$ & 1.1 (1 to 1.3$)$ & 1.1 (1 to 1.3$)$ & 0.3 \\
\hline $\mathrm{RBC} / 24 \mathrm{~h}$ & $10.99 \%$ & $4.71 \%$ & $15.18 \%$ & 0.87 \\
\hline r-TEG ACT (s) & 121 (113 to 128$)$ & $121(113$ to 136$)$ & $121(113$ to 128$)$ & 0.25 \\
\hline r-TEG angle (deg) & $72.7(67.5$ to 76.1$)$ & $70.6(66$ to 74.7$)$ & $70.6(67$ to 74.8$)$ & 0.29 \\
\hline r-TEG MA (mm) & 62.5 (58 to 67$)$ & $63.3(56.5$ to 66.5$)$ & $61.5(56.5$ to 65.5$)$ & 0.36 \\
\hline r-TEG LY30 (\%) & 2.3 (1.3 to 3.7 ) & $2.2(1.4$ to 3.4$)$ & $1.2(0.6$ to 2.7$)$ & $<0.001$ \\
\hline Length of stay (LOS) & $5(2$ to 11$)$ & $5.5(1.5$ to 13$)$ & 7 (3 to 14$)$ & 0.22 \\
\hline VFD & 26 (22 to 28$)$ & 27 (24.5 to 28$)$ & 26 (23 to 28$)$ & 0.85 \\
\hline ICUFD & 24 (20 to 28$)$ & $25(21.5$ to 28$)$ & 25 (17 to 27$)$ & 0.87 \\
\hline
\end{tabular}

Continuous variables are presented as median and interquartile range while categorical variables are presented as percent

The ${ }^{*} \chi^{2}$ test was used for categorical variables and Kruskal-Wallis test for continuous variables

$B A L$ blood alcohol level, TBI traumatic brain injury, BMI body mass index, NISS New Injury Severity Score, $E D$ emergency department $S B P$ systolic blood pressure, $H R$ heart rate, GCS Glasgow Coma Scale, INR International Normalized Ratio, TEG thrombelastography, $A C T$ activated clotting time, $M A$ maximum amplitude, $L Y 30$ lysis $30 \mathrm{~min}$ after MA is achieved, VFD ventilator-free days, ICUFD intensive care unitfree days had BAL measured seemed slightly more likely to have TBI but had less bleeding/shock than those that were not tested for BAL. This could be explained by the fact that alcohol intoxication suppresses cognitive and motor function resulting in a lower GCS.

Several studies have investigated the potential role of alcohol consumption and thromboembolic risk. Spoerke et al. [23] showed that both males and females had increased PAI-1, the inhibitor of tPA-mediated fibrinolysis, after consumption of alcohol. In the male population, this was also associated with a small decrease in LY30 (clot breakdown). In a study evaluating the effects of alcohol consumption on cardiac risk, Djousse et al. [9] found that individuals who consumed larger amounts of alcohol, more than $14.9 \mathrm{~g}$ of alcohol per day (the equivalent of one drink), had increased circulating PAI-1, which they postulated increased the risk of thrombotic complications. Furthermore, evidence supports that in acute alcohol intoxication, there is an increase in both tPA and PAI-1 [11, 12]. However, the ratio of PAI$1:$ tPA is shifted to favor PAI-1 with the consumption of an 
Table 3 Correlations with rapid TEG parameters and blood alcohol level

\begin{tabular}{lcr}
\hline & \multicolumn{2}{l}{ BAL } \\
\cline { 2 - 3 } & Rho & $p$ value \\
\hline rTEG ACT & -0.119 & 0.10 \\
rTEG Angle & -0.093 & 0.20 \\
rTEG MA & -0.101 & 0.17 \\
rTEG LY30 & -0.315 & $<0.001$ \\
\hline
\end{tabular}

Inverse correlations between blood alcohol level and LY30 on rTEG were seen. Data are presented as Spearman correlation coefficient (Rho). Statistical significance is $p<0.05$

rTEG rapid thrombelastography, $A C T$ activated clotting time, $M A$ maximum amplitude, $L Y 30$ fibrinolysis $30 \mathrm{~min}$ after MA is achieved

increased amount of alcohol [12]. Furthermore elevated triglyceride levels resulting from heavy alcohol consumption may further stimulate PAI-1 gene expression, especially in people with a genetic makeup particularly sensitive to PAI-1 [24]. This increased PAI-1 gene expression could result in the inhibition of fibrinolysis and thus increase the risk for acute cardiac events [24]. Interestingly, the type of alcohol consumed appears to effect the ratio of PAI-1:tPA. Tousoulis et al. investigated the effects of types of alcohol on the fibrinolytic system and discovered that acute alcohol consumption increased the PAI-1:tPA ratio; however, this effect was not observed after the consumption of red wine [25]. These data support the notion that not only the amount of alcohol consumed but the type may adversely affect cardiovascular health.

In our study, a higher BAL $(>150 \mathrm{mg} / \mathrm{dL}$ or $>1.5 \mathrm{~g} / \mathrm{L})$ was associated with a lower LY30 on r-TEG suggesting increased resistance to tPA in this population. Furthermore, the odds of fibrinolysis shutdown were threefold higher if the BAL was $>150 \mathrm{mg} / \mathrm{dL}(>1.5 \mathrm{~g} / \mathrm{L})$ and this BAL was also an independent predictor of fibrinolysis shutdown with an AUROC of 0.76 , indicating BAL $>150 \mathrm{mg} / \mathrm{dL}(>1.5 \mathrm{~g} / \mathrm{L})$ is a fair predictor of fibrinolysis shutdown. In vitro and in vivo studies have provided data that ethanol affects the fibrinolysis profile and studies in healthy human volunteers suggest that this decrease in fibrinolysis is secondary to
Fig. 1 Blood alcohol level (BAL) and fibrinolysis phenotypes ( $S D$ fibrinolysis shutdown, HYPER hyperfibrinolysis, $P H Y$ physiologic). Patients with a high BAL class had an increased incidence of fibrinolysis compared to those with no detectable blood alcohol and those with $>0-150 \mathrm{mg} /$ $\mathrm{dL}(0-1.5 \mathrm{~g} / \mathrm{L})$

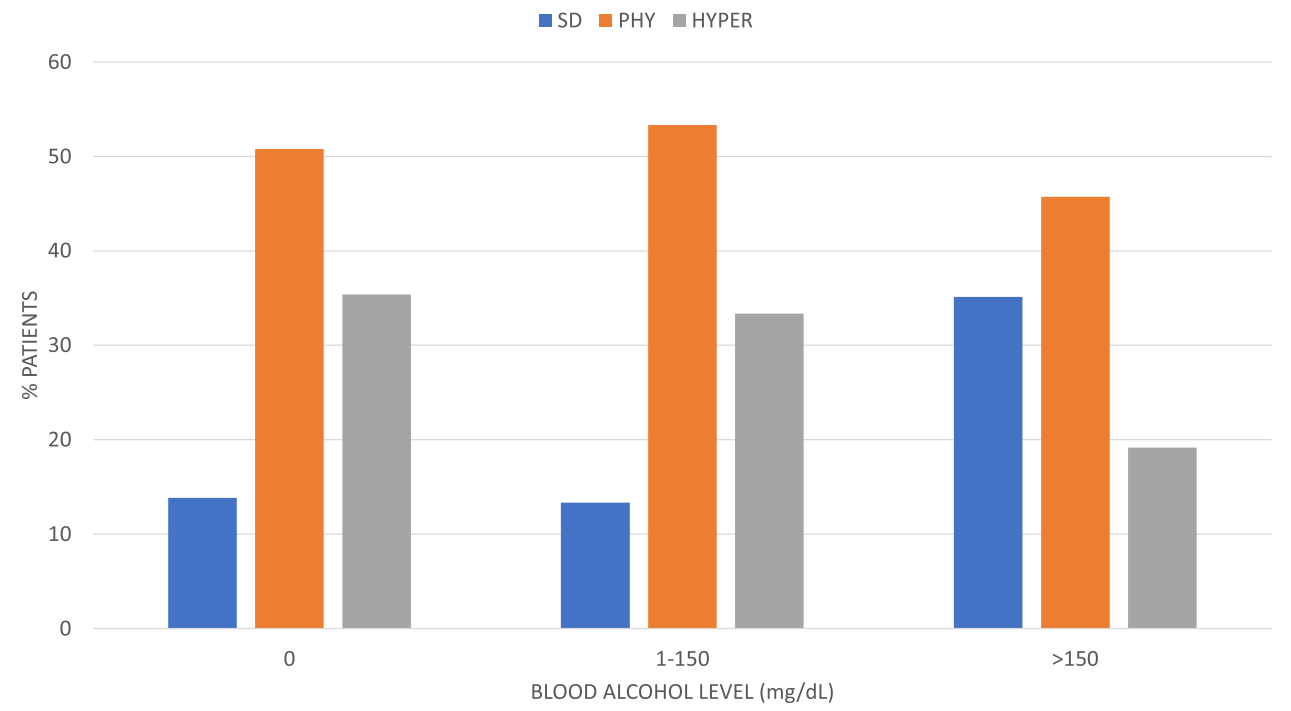

\begin{tabular}{llllll}
\hline Dependent variable & BAL $(\mathrm{mg} / \mathrm{dL})$ & Odds ratio & \multicolumn{2}{l}{$95 \%$ confidence limits } & $p$ value \\
\hline Hyperfibrinolysis & $>150$ vs $<10$ & 0.501 & 0.214 & 1.171 & 0.11 \\
& $>150$ vs $10-150$ & 0.827 & 0.297 & 2.301 & 0.72 \\
Shutdown & $10-150$ vs $<10$ & 0.606 & 0.215 & 1.708 & 0.34 \\
& $>150$ vs $<10$ & 3.025 & 1.11 & 8.244 & 0.03 \\
& $>150$ vs $10-150$ & 4.18 & 1.047 & 16.683 & 0.043 \\
& $10-150$ vs $<10$ & 0.724 & 0.151 & 3.464 & 0.69 \\
\hline
\end{tabular}

Multinomial logistic regression testing the independent effect of blood alcohol level (BAL) on a three-category outcome: hyperfibrinolysis (HYPER), fibrinolysis shutdown (SD) and physiologic fibrinolysis serving as the reference group. Odds ratios are adjusted for age, blunt mechanism, NISS, admission GCS and SBP. Deviance $p=0.674$ and Pearson goodness-of-fit test $p=0.156$ (higher $p$ values indicate better goodness-offit) 
circulating levels of PAI-1 $[2,26]$. Our data are consistent with other previously published data that high levels of alcohol intoxication are associated with decreased fibrinolytic activity while low level of alcohol intoxication is not [9-12]. Furthermore, these data confirm that of Howard et al. who also recently evaluated alcohol effects on fibrinolysis using ROTEM [27]. They similarly illustrated that elevated levels of ETOH resulted in decreased fibrinolysis by ROTEM [27]. The confirmation of impaired fibrinolysis, on two similar but different viscoelastic platforms, in these two separate patient populations strengthens the notion that acute alcohol intoxication influences fibrinolytic phenotype more than previously thought and could be a risk factor for the development of fibrinolysis shutdown-related adverse outcomes.

Several studies have evaluated distinct fibrinolysis phenotypes, with fibrinolysis shutdown being associated with increased mortality and rates of organ failure $[15,17,28]$. The CRASH-2 trial suggested that the early empiric use of tranexamic acid (TXA) reduces the rate of death in injured patients but identified increased mortality when this therapy was delivered $>3 \mathrm{~h}$ after injury [29]. Our group has argued that there should be a selective use of TXA in the injured patient as administration to those with a shutdown phenotype may be critical in the pathogenesis of postinjury organ failure and thrombotic complications [30]. At our institution, TXA is administered to patients that are in hemorrhagic shock with evidence of hyperfibrinolysis on TEG as we have found that the use of TXA in those not in hyperfibrinolysis may increase the risk of postinjury organ failure, thrombotic complications, and mortality $[17,30]$. Experimental data have revealed that ethanol-exposed animals show enhanced PAI-1 expression and pulmonary fibrin deposition with coincident exaggeration of pulmonary edema and inflammatory injury [31]. High levels of alcohol and subsequent excess expression of PAI-1 may thus be a contributing factor toward the development of organ failure following injury and would likely be exacerbated with the concomitant use of antifibrinolytic therapy. Less than $10 \%$ of trauma activation patients received antifibrinolytic therapy overall, and less than $5 \%$ in patients that had a BAL measured making subgroup analysis of patients that received TXA unreliable.

However, while our data reveal increased odds of fibrinolysis shutdown in acutely intoxicated patients, there is an absence of increased mortality in this group as we would expect for those patients in fibrinolysis shutdown. This is also seen in other studies evaluating alcohol's effects on fibrinolysis [27]. This could be due to an underpowered study to identify mortality differences. Furthermore, once the acute episode of intoxication resolves, the fibrinolytic phenotype could change from that of fibrinolysis shutdown to physiologic fibrinolysis or even hyperfibrinolysis. It may be that the patients who remain in a phenotype of fibrinolysis shutdown after the resolution of acute alcohol intoxication are the ones that have adverse effects in the form of late mortality or MOF.

Identifying patients with a high BAL would allow the stratification of patients into those with a higher risk of having fibrinolysis shutdown. Low fibrinolytic activity has been associated with an increased mortality following injury, with a mortality of $17-22 \%$ compared to a physiologic level of fibrinolysis of $3-14 \%[15,28]$. The cause of death in the shutdown cohort was typically late death as previously discussed, and more commonly associated with multiorgan failure $[15,28]$. It has been a hypothesis that multiple organ failure is caused by microthrombotic complications stemming from the decreased clot breakdown associated with fibrinolysis shutdown $[15,28]$. There are no pharmacologic interventions currently in use to specifically target fibrinolysis shutdown. However, by identifying alcohol intoxication as a modulator of the fibrinolysis phenotype, this could help guide future interventions to help reduce the incidence of fibrinolysis shutdown and subsequent micro- and macrothormbotic complications in the critically injured patient. Data suggest that an increased amount of PAI-1 and shifting the tPA:PAI-1 ratio in favor of PAI-1 is a likely factor that contributes toward increased incidence of fibrinolysis shutdown in the intoxicated patient.

The presented study has some limitations. These data reflect a single point in time of a dynamic process and does not take into account the temporal changes of the coagulation process and subsequent evolution of fibrinolysis phenotypes. Unfortunately, there were patients in our TAP protocol that could not be included in the study because no BAL was obtained. BAL was obtained at the discretion of the trauma team. This may have led to a selection bias that must be taken into consideration with the interpretation of these results. Furthermore, we did not have information on the amount or type of alcohol ingested, the time during which alcohol was consumed, and reliable data on chronic ingestion of alcohol, which may have diverse effects. There were very few patients within our analyzed cohort that had documentation of chronic alcoholism $(n=26,13.6 \%)$. There was a statistically significant trend (M-H Chi-sq, $p=0.019)$ of high a BAL with documentation of chronic alcoholism, which may be due to increased frequency of documentation among BAL-positive patients. The significant association between BAL levels and LY30 was detected again among patients without documentation of chronic alcoholism $(p=0.007)$. However, the small number of patients with documented alcoholism limits the analysis in this group and, therefore, for the purpose of this study, we did not separate acute vs chronic users for analysis. We also acknowledge that a high BAL leading to increased risk of VTE is based on theory at this time as our cohort was not large enough to appropriately study this question. 
In conclusion, in the injured patient, high BAL $(>150 \mathrm{mg} / \mathrm{dL}$ or $>1.5 \mathrm{~g} / \mathrm{L})$ is an independent predictor for fibrinolysis shutdown. The mechanism of alcohol-related shutdown remains to be elucidated however, data would suggest a shift in the PAI-1:tPA ratio favoring PAI-1 may be a major contributor. Postinjury fibrinolysis shutdown has been associated with increased risk of organ failure and thrombotic complications; thus, a high BAL may represent a risk factor for VTE and microthrombotic events. Furthermore, the use of anti-fibrinolytic agents in patients with high BAL may have adverse effects by precipitating a more profound resistance to fibrinolysis.

Author contributions GRS implemented the study, interpreted data, drafted and critically revised the manuscript. HBM interpreted data, drafted, and critically revised the manuscript. GRN interpreted data, drafted, and critically revised the manuscript. BJH interpreted data, drafted, and critically revised the manuscript. EEM, CCS, AB, and AS are principal investigators and were responsible for study conception and design, implementation of study, completion of study, interpretation of data, manuscript drafting, and critical revision.

\section{Compliance with ethical standards}

Conflict of interest Gregory R Stettler MD declares that he has no conflict of interest. Ernest E Moore MD declares that he has no conflict of interest. Geoffrey R Nunns MD declares that he has no conflict of interest. Hunter B Moore MD declares that he has no conflict of interest. Benjamin R Huebner MD declares that he has no conflict of interest. Christopher C Silliman MD, PhD, serves on the Scientific Advisory Board for Hemanext ${ }^{\mathrm{TM}}$. Anirban Banerjee $\mathrm{PhD}$ declares that he has no conflict of interest. Angela Sauaia MD, PhD, declares that he has no conflict of interest.

Ethical standards Research reported in this publication was supported in part by the National Institute of General Medical Sciences Grants: T32-GM008315 and P50-GM49222, the National Heart Lung and Blood Institute UM1-HL120877, in addition to the Department of Defense USAMRAA and W81XWH-12-2-0028. The content is solely the responsibility of the authors and does not necessarily represent the official views of the National Institutes of Health, the National Heart, Lung, and Blood Institute, or the Department of Defense. Additional research support was provided by Haemonetics with shared intellectual property.

Studies involving human participants All procedures performed in studies involving human participants were in accordance with the ethical standards of the institutional and/or national research committee (COMIRB \#13-3087) and with the 1964 Helsinki declaration and its later amendments or comparable ethical standards. Informed consent was obtained from all individual participants or their surrogates included in the study.

\section{References}

1. Moore EE. Alcohol and trauma: the perfect storm. J Trauma. 2005;59(3 Suppl):S53-S5656 (discussion S67-75).

2. Spoerke N, Underwood S, Differding J, et al. Effects of ethanol intoxication and gender on blood coagulation. J Trauma.
2010;68(5):1106-11. https://doi.org/10.1097/TA.0b013e3181 d86860.

3. Howard BM, Kornblith LZ, Redick BJ, et al. The effects of alcohol on coagulation in trauma patients: interpreting thrombelastography with caution. J Trauma Acute Care Surg. 2014;77(6):865-71. https://doi.org/10.1097/TA.0000000000000357(discussion 71-2).

4. Cook MR, Louis SG, McCully SP, et al. Positive blood alcohol is associated with reduced DVT in trauma. Injury. 2015;46(1):1315. https://doi.org/10.1016/j.injury.2014.09.017.

5. Phelan H, Stahls P, Hunt J, et al. Impact of alcohol intoxication on hemodynamic, metabolic, and cytokine responses to hemorrhagic shock. J Trauma. 2002;52(4):675-82.

6. van de Wiel A, van Golde PM, Kraaijenhagen RJ, et al. Acute inhibitory effect of alcohol on fibrinolysis. Eur J Clin Invest. 2001;31(2):164-70.

7. Neupane SP, Skulberg A, Skulberg KR, et al. Cytokine changes following acute ethanol intoxication in healthy men: a crossover study. Mediators Inflamm. 2016;2016:3758590. https://doi. org/10.1155/2016/3758590.

8. Yeligar SM, Chen MM, Kovacs EJ, et al. Alcohol and lung injury and immunity. Alcohol. 2016;55:51-9. https://doi.org/10.1016/j. alcohol.2016.08.005.

9. Djousse L, Pankow JS, Arnett DK, et al. Alcohol consumption and plasminogen activator inhibitor type 1: the National Heart, Lung, and Blood Institute Family Heart Study. Am Heart J. 2000;139(4):704-9.

10. Marques-Vidal P, Cambou JP, Nicaud V, et al. Cardiovascular risk factors and alcohol consumption in France and Northern Ireland. Atherosclerosis. 1995;115(2):225-32.

11. Veenstra JtW E, Kluft C. Alcohol and fibrinolysis. Fibrinolysis. 1990;4(Supp 2):64-8.

12. Dimmitt SB, Rakic V, Puddey IB, et al. The effects of alcohol on coagulation and fibrinolytic factors: a controlled trial. Blood Coagul Fibrinol Int J Haemost Thromb. 1998;9(1):39-45.

13. Gorelick PB. Alcohol and stroke. Stroke. 1987;18:268-71.

14. Mukamal KJ, Jadhav PP, D'Agostino RB, et al. Alcohol consumption and hemostatic factors: analysis of the Framingham Offspring cohort. Circulation. 2001;104(12):1367-73.

15. Moore HB, Moore EE, Liras IN, et al. Acute fibrinolysis shutdown after injury occurs frequently and increases mortality: a multicenter evaluation of 2540 severely injured patients. J Am Coll Surg. 2016;222(4):347-55. https://doi.org/10.1016/j.jamco 1lsurg.2016.01.006.

16. Meizoso JP, Karcutskie CA, Ray JJ, et al. Persistent fibrinolysis shutdown is associated with increased mortality in severely injured trauma patients. J Am Coll Surg. 2017;224(4):575-82. https://doi.org/10.1016/j.jamcollsurg.2016.12.018.

17. Moore EE, Moore HB, Gonzalez E, et al. Postinjury fibrinolysis shutdown: rationale for selective tranexamic acid. J Trauma Acute Care Surg. 2015;78(6 Suppl 1):S65-S6969. https://doi. org/10.1097/TA.0000000000000634.

18. Kashuk JL, Moore EE, Sawyer M, et al. Postinjury coagulopathy management: goal directed resuscitation via POC thrombelastography. Ann Surg. 2010;251(4):604-14. https://doi.org/10.1097/ SLA.0b013e3181d3599c.

19. Wohlauer MV, Moore EE, Thomas S, et al. Early platelet dysfunction: an unrecognized role in the acute coagulopathy of trauma. J Am Coll Surg. 2012;214(5):739-46. https://doi.org/10.1016/j. jamcollsurg.2012.01.050.

20. Gonzalez E, Pieracci FM, Moore EE, et al. Coagulation abnormalities in the trauma patient: the role of point-of-care thromboelastography. Semin Thromb Hemost. 2010;36(7):723-37. https:// doi.org/10.1055/s-0030-1265289.

21. Einersen PM, Moore EE, Chapman MP, et al. Rapid thrombelastography thresholds for goal-directed resuscitation of patients at risk for massive transfusion. J Trauma Acute Care Surg. 
2017;82(1):114-9. https://doi.org/10.1097/TA.000000000000127 0 .

22. Moore HB, Moore EE, Gonzalez E, et al. Plasma is the physiologic buffer of tissue plasminogen activator-mediated fibrinolysis: rationale for plasma-first resuscitation after life-threatening hemorrhage. J Am Coll Surg. 2015;220(5):872-9. https://doi. org/10.1016/j.jamcollsurg.2015.01.026.

23. Macko AR, Moore H, Cap AP, et al. Tissue injury suppresses fibrinolysis following hemorrhagic shock in non-human primates (rhesus macaque). J Trauma Acute Care Surg. 2017;82:4. https:// doi.org/10.1097/TA.0000000000001379.

24. Zakhari S. Alcohol and the cardiovascular system: molecular mechanisms for beneficial and harmful action. Alcohol Health Res World. 1997;21(1):21-9.

25. Tousoulis D, Ntarladimas I, Antoniades C, et al. Acute effects of different alcoholic beverages on vascular endothelium, inflammatory markers and thrombosis fibrinolysis system. Clin Nutr. 2008;27(4):594-600. https://doi.org/10.1016/j.clnu.2008.01.002.

26. Engstrom M, Schott U, Reinstrup P. Ethanol impairs coagulation and fibrinolysis in whole blood: a study performed with rotational thromboelastometry. Blood Coagul Fibrinol Int J Haemost Thromb. 2006;17(8):661-5. https://doi.org/10.1097/MBC.0b013 e32801010b7.
27. Howard BM, Kornblith LZ, Redick BJ, et al. Exposing the bidirectional effects of alcohol on coagulation in trauma: impaired clot formation and decreased fibrinolysis in rotational thromboelastometry. J Trauma Acute Care Surg. 2018;84(1):97-103. https ://doi.org/10.1097/TA.0000000000001716.

28. Moore HB, Moore EE, Gonzalez E, et al. Hyperfibrinolysis, physiologic fibrinolysis, and fibrinolysis shutdown: the spectrum of postinjury fibrinolysis and relevance to antifibrinolytic therapy. J Trauma Acute Care Surg. 2014;77(6):811-7. https://doi. org/10.1097/TA.0000000000000341(discussion 17).

29. Roberts I, Shakur H, Coats T, et al. The CRASH-2 trial: a randomised controlled trial and economic evaluation of the effects of tranexamic acid on death, vascular occlusive events and transfusion requirement in bleeding trauma patients. Health Technol Assess. 2013;17(10):1-79. https://doi.org/10.3310/hta17100.

30. Moore EE, Moore HB, Gonzalez E, et al. Rationale for the selective administration of tranexamic acid to inhibit fibrinolysis in the severely injured patient. Transfusion. 2016;56(Suppl 2):S110 S11414. https://doi.org/10.1111/trf.13486.

31. Poole LG, Massey VL, Siow DL, et al. Plasminogen activator inhibitor- 1 is critical in alcohol-enhanced acute lung injury in mice. Am J Respir Cell Mol Biol. 2017;57(3):315-23. https://doi. org/10.1165/rcmb.2016-0184OC. 